\title{
Chaos Control in Chaotic Dynamical Systems Via Auto-tuning Hamilton Energy Feedback
}

\author{
A. Reza Ahrabi ${ }^{1, *}$, H. Kobravi ${ }^{2}$ \\ ${ }^{1}$ Islamic Azad University, Faculty of Engineering, Department of Electrical Engineering, 9187147578 Mashhad, Iran \\ ${ }^{2}$ Islamic Azad University, Faculty of Medical Engineering, Research Center of Biomedical, 9187147578 Mashhad, Iran
}

\section{A R T I C L E I N F O}

Article history:

Received $\quad 17$ December 2019

Accepted $\quad 31$ December 2019

Available online 31 December 2019

\section{Keywords:}

Hamilton energy

Chaos control

Fuzzy logic system

\begin{abstract}
A B S T R A C T
The converting of an unwelcome chaotic behaviour of the brain activity into another chaotic behaviour can result in some disorders and their treatment as an epileptic seizure. Recently in some articles, energy-based feedback control is introduced only as an aim to omit the chaos. In other words, it is used only for anti-control of chaos. But in this study, the capability of changing chaotic dynamics to other ones with a feedback energy-based controller has been demonstrated. The converting of chaos dynamics to another chaos dynamics can be made possible by using energy feedback and Limiting to omit the chaos should not be occurred by using energy feedback. Some practical applications of chaos to chaos controllable related to the emphasis of this issue. A short glance at a well-known Lorenz chaotic system has been indicated that chaos to chaos control will be reachable by combining a self-regulating gain fuzzy system with an energy feedback control, as well.
\end{abstract}

(đ) (ब ) 2019 Turkish Journal of Forecasting by Giresun University, Forecast Research Laboratory is licensed under a Creative Commons Attribution-ShareAlike 4.0

\section{Introduction}

The study of chaos control has been engaged by many researchers in recent years, because of its practical applications such as in laser physics, secure communications, nonlinear circuits, systems modelling, and in power, electrical, biological and mechanical systems $[1,2,3,4,5,6,7,8]$. The researchers related to chaos control can be classified into 3 categories, those who focus on the synchronization of chaos $[9,10,11]$, on the regulation of chaos [12] and on the tracking control of chaos [13,14,15].

In particular, the research related to tracking control of the chaotic system itself focuses on two different classes. One of these two classes is where the system output is directed to a specific chaotic output $[9,13,14,15,16]$. Another category of chaotic system tracking is that anti control of the chaos. In fact, researchers try to direct unpleasant chaos to another unwilling chaos called anti control of the chaos that few articles are found in this area $[17,18,19]$. One of the methods related to anti control of chaos is the well-known OGY method. In a chaotic system, trajectories will not be able to move to an unstable predicted orbit or, if successful, will be eliminated rapidly. By the use of the OGY method, the trajectories of the chaotic system are forced to remain on the same unstable orbit for a short time. In 
other words, the OGY method is a discrete technique that considers small perturbations promoted in the neighbourhood of the desired orbit when the trajectory crosses a specific surface [20].

But some disorders and their treatment such as epileptic seizure can be shown by converting an unwilling chaotic behaviour of the brain activity into another chaotic behaviour. It shows the necessity of applying such approaches of chaos to chaos control in order to control the biological systems. For instance, during epileptic seizures' episodes, there are some bifurcations to states system can be proven [4,5]. We can say that after epileptic seizures, geometric features of the basin of attraction for the EEG signal change [6]. Hence, we can consider that converting an unwelcome chaotic dynamic into some other chaotic dynamical regime, would be possible by the treatment of such disorders.

Because of the complexity of chaotic systems, most of the methods were mentioned for a particular class of chaotic systems either only control a fixed stable point or their synchronization, tracking, and regulation. In fact, the antichaos control factor is the limiting factor that drives the system to another desirable chaos by exerting pressure on the system dynamics. However, there is no mention of the important issue of converting chaotic dynamics into desirable chaotic dynamics. Since in biological systems, accessibility of the system parameters and system input are practically difficult or impossible [4], direct or indirect control of chaotic behaviour is an open research issue. As a result, chaos dynamics correction is necessary but has not been addressed in the methods described in classical control.

Since changing the system's dynamics during the time can be presented by the Hamilton energy function, this has drawn the attention of many sciences in recent decades [1,2]. Nowadays we witness that using the Hamiltonian energy in the chaos control of dynamical systems has been prevalent [1]. The ability to suppress the chaos in periodic oscillators and hyper-chaotic systems by using the suggested method, via the energy modulation and negative feedback of the Hamilton function, would be possible [1]. However, a control scheme based on the energy feedback, in this study has been seen that via applying the online regulation of energy feedback gain, converting chaotic dynamics into chaotic dynamics would be made possible. Design of such approaches in control of biological systems, this can be important enough in the realm of the practical point of view [3].

It is clear that, in the state space, for chaos to chaos controlling, energy changes can be expressive of the changes in the pattern of the basin of attraction, hence, it seemed controller of energy-based feedback can also be viable. So, in this research, we illustrate the potential of the mentioned control strategy for chaos to chaos control.

This paper is organized as follows. In the present section, a brief introduction related to the literature survey was expressed. Methods and materials description and proposed control strategy are introduced in Section 2. The Results, simulations, and discussion are addressed in Section 3 and The Lorenz chaotic system is taken as an example to show the feasibility and efficiency of theoretical results. Finally, the conclusion is provided in Section 4.

\section{Methods and Materials}

\subsection{The proposed control strategy}

As we just mentioned in the introductory section of the article, we are looking for a quantity that can describe the pattern of attractors and energy can be a proper quantity to describe the pattern of attractors because changes in energy over time reflects the change in the patterns of the attractor $[21,22]$.

\subsubsection{Hamilton energy function-based controller}

One of the suggestions that has come up in recent years is a quantity called Hamilton Energy. Hamilton Energy is a quantity that corresponds to the sum of the total system energy and represents kinetic energy and potential energy. By Helmholtz's theorem, we break down any velocity vector field $f(x)$ into a sum of two vector fields, one divergencefree vector $f_{c}(x)$ that calculates rotational tensor of $f(x)$ and the other one is one gradient vector field $f_{d}(x)$ which carries its divergence [22]

$f(x)=f_{c}(x)+f_{d}(x)$

where $\mathrm{f}(\mathrm{x})$ is the general autonomous dynamical system. Eq.2 can be used to specify the energy of the system. the generalized Hamiltonian form for dynamical equations that plays the role of the system's total energy, is

$\frac{d X}{d t}=[J(x)+R(x)] \nabla H$ 
where $\nabla \mathrm{H}, J(x)$ and $R(x)$ are the gradient vector of a smooth energy function $H(x)$, a skew-symmetric matrix and asymmetric matrix, respectively.

\subsubsection{Controller design negative Hamilton energy feedback}

Since in chaotic systems, the desired trajectory is not definable and Since in biological systems, we do not have access to dynamic system parameters or input system, we want to modify the system dynamics by using external feedback so that we can change the attractor pattern by the energy feedback controller and convert chaos dynamics into desired chaos dynamics. The negative feedback that is proportional to the Hamiltonian function has been applied to energy as follows:

$\dot{H}=\nabla H^{T} f_{d}(r) \nabla H-\mathrm{kH}$

where -KH is negative feedback Hamilton energy and K is Hamilton energy's Gain. Hamilton feedback is observed feedback from the system that in additions to having information on system output, also has lots of information on system behaviour and its dynamics. Indeed, by adding Eq.3 to the Lorenz system equations, we indirectly not as system input, change the attractor's pattern by negative feedback Hamilton energy.

Our proposal is that if by using an online self-regulating system, the gain control of feedback is run, the ability for controlling with Hamilton's feedback would not be bounded to the chaos omission. Since we intend to set the parameter $\mathrm{k}$ by the fuzzy controller, a fuzzy system will be appropriated whose then-part is a number. The rules written for the Takagi-Sugeno fuzzy system are numerals instead of membership functions, which are therefore easy to implement and are suitable for tuning the energy feedback. So, we apply a Takagi-Sugeno fuzzy logic system in the control system as an online regulator controller. We set the fuzzy logic system's input as an amount of states' distance from the origin and the speed of its changes. the output of the fuzzy system shows the amount of energy feedback gain. In order to stay limited the minimum distance of states from the origin and the speed of their variations from the origin, fuzzy rules have been extracted. So, the stability of the control system is also guaranteed by changing the parameters of the fuzzy system. The fuzzy set of inputs is limited to $[-0.5,0.5]$ and their input values are both $\{\mathrm{N}$, $\mathrm{Z}, \mathrm{P}\}$ which are negative, zero and positive, respectively. The output, energy feedback gain, is limited to $\{0,1\}$. Triangular membership functions are used as Inputs when the amount of states' distance from the origin is in $\mathrm{N}$ and the speed of its change is N, according to trial and error, it indicates that the amount of energy feedback gain, should be at the minimum value. The results of our circumstances can be driven similarly.

\section{Results and Discussion}

We appraised the usefulness of the suggested strategy for various initial conditions. Figure 1 illustrates the membership functions of inputs of the fuzzy system, and Table 1 illustrates the rules which are applied for the fuzzy system. When the controller was activated, the system behaviour changed after a transitory period. The third-order continuous-time well-known Lorenz chaotic system which based on a nonlinear and chaotic behaviour, described by [22]:

$$
\begin{aligned}
& \dot{x_{1}}=\sigma\left(x_{2}-x_{1}\right) \\
& \dot{x_{2}}=\beta x_{1}-x_{2}-x_{1} x_{3} \\
& \dot{x_{3}}=-\rho x_{3}+x_{1} x_{2}
\end{aligned}
$$

where parameters $\sigma, \beta$ and $\rho$ are system parameters which in this study we used $\sigma=10, \beta=\frac{8}{3}, \rho=28$.

According to Eq. (2), $f_{d}=\left(\begin{array}{c}-\sigma x \\ -y \\ -\beta z\end{array}\right)$ is the responsible part for the divergence of the field and $\mathrm{f}_{\mathrm{c}}=\left(\begin{array}{c}\sigma \mathrm{y} \\ \rho \mathrm{x}+\mathrm{xz} \\ \mathrm{xy}\end{array}\right)$ is the part that does not contribute to it and by using Eq. (2), the Hamilton equation of Lornz system is as follows [22]

$H_{\text {Lorenz }}(x \cdot y \cdot z)=\frac{1}{2}\left(-\frac{\rho}{\sigma} x^{2}+y^{2}+z^{2}\right)$

and Hamilton energy derivative is:

$\dot{H}_{\text {Lorenz }}(x \cdot y \cdot z)=\rho x^{2}-y^{2}-\beta z^{2}$ 
For example, in the presence of a controller and without the controller for the initial conditions $(0.2,0.1$ and 0.2$)$, the trajectories in the state space of Lorenz chaotic system is shown in Figure 2. We can use Wolf's well-known method to calculate the values of Lyapunov spectrums for investigating chaos dynamic changes in the System.

We use for initial conditions [27], the Averaged Lyapunov spectrums, where $\lambda_{n}$ is the Lyapunov exponent in the $n^{\text {th }}$ initial condition and $i$ is considered as the number of initial conditions, as follows:

$\bar{\lambda}=\frac{1}{i} \sum_{n=1}^{i} \lambda_{n}$

We can see the results of calculating the Averaged Lyapunov Exponents in the presence of a controller and without it on Table 2.

Table 1. Rules of fuzzy extracted in Takagi-Sugeno fuzzy system

\begin{tabular}{cccc}
\hline Edot E & Negative & Zero & Positive \\
\hline Negative & 0.01 & 0.7 & 0.2 \\
Zero & 1 & 0.01 & 0.5 \\
Positive & 0.5 & 1 & 0.7 \\
\hline
\end{tabular}

According to the results (Table 2), the difference in Lyapunov spectrums in the uncontrolled mode and with the presence of the controller mode were:

$$
\begin{aligned}
& \left|\overline{\lambda_{x-\text { посо }}}-\overline{\lambda_{x-\text { wсo }}}\right|=0.1259 \\
& \left|\overline{\lambda_{y-\text { noco }}}-\overline{\lambda_{y-\text { wco }}}\right|=0.10048 \\
& \left|\overline{\lambda_{z-\text { noco }}}-\overline{\lambda_{z-w c o}}\right|=0.114
\end{aligned}
$$

Table 2. The computing values of averaged Lyapunov exponents calculated after applying the controller and before applying the controller $\left(\sigma=10, \beta=\frac{8}{3} \cdot \rho=28\right)$

\begin{tabular}{cc}
\hline Uncontrolled system & Controlled system \\
\hline$\overline{\lambda_{x}}=0.05044$ & $\overline{\lambda_{x}}=0.17634$ \\
$\overline{\lambda_{y}}=0.05922$ & $\overline{\lambda_{y}}=0.15970$ \\
$\overline{\lambda_{z}}=0.04232$ & $\overline{\lambda_{z}}=0.15642$ \\
\hline
\end{tabular}

The observed variations of the Lyapunov spectrum mean that using the controller gave rise to changing the system dynamics and pattern of the system attractor in the state space. In fact, through exerting the controller, the system trajectories gradually entered a new attractor showing the system dynamics converted to some new dynamics.
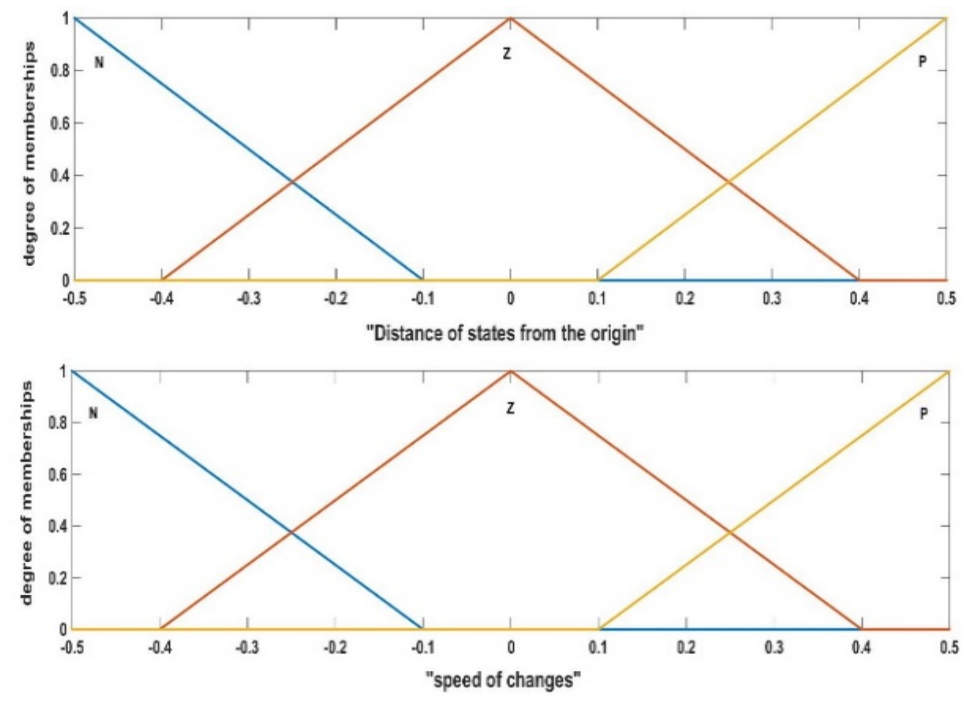

Figure 1. Fuzzy membership functions of the fuzzy inputs system 


\section{Conclusion}

A new argument is raised that instead of anti-chaos control methods, we drive system dynamics from undesirable chaos to desirable ones. But it is mandatory to use closed-loop control methods which is a feedback controller. As we know, the classic feedback controller, only relevant to states or output system feedback. For converting an unwilling chaos dynamic to the desired chaos dynamics, we had to define special feedback that comes from a quantity whose changes over time reflect changes in chaos dynamics and also includes a parameter describing system dynamics. One of the suggestions that have come up in recent years is to get feedback from a quantity called Hamilton, which represents the energy of the system itself. Hamilton's feedback is observed from a system that, in addition to having information on system output, also has much information on system behaviour and dynamics. The main idea in this study is to use Hamilton's feedback with a fuzzy self-regulating system that could be able to enhance the capabilities of the control system so that it could be used both to control chaos to desired chaos and to regulate the chaos.

It can be understood by the results that we can use the fuzzy self-regulating system in order to convert chaotic dynamics into another one by maintaining the control system's stability. Thus, it can be shown that chaos to chaos control can be made feasible by using mechanisms of online optimization to control energy feedback. The difference in Lyapunov spectrums in both uncontrolled and controlled mode of the chaotic Lorenz system means that the attractor pattern has changed, and trajectories of the system showing the system dynamics converted to some new dynamics. We believe in the point that never we can talk about the similarity of system dynamics by comparing peakto-peak indices. The application of this controlling attitude should not be limited to delete chaos. A widespread of horizons for researchers are available and would encourage engineers and researchers in the realm of chaos control not too distant future and we want to test this control method on other chaotic systems and by real data too.
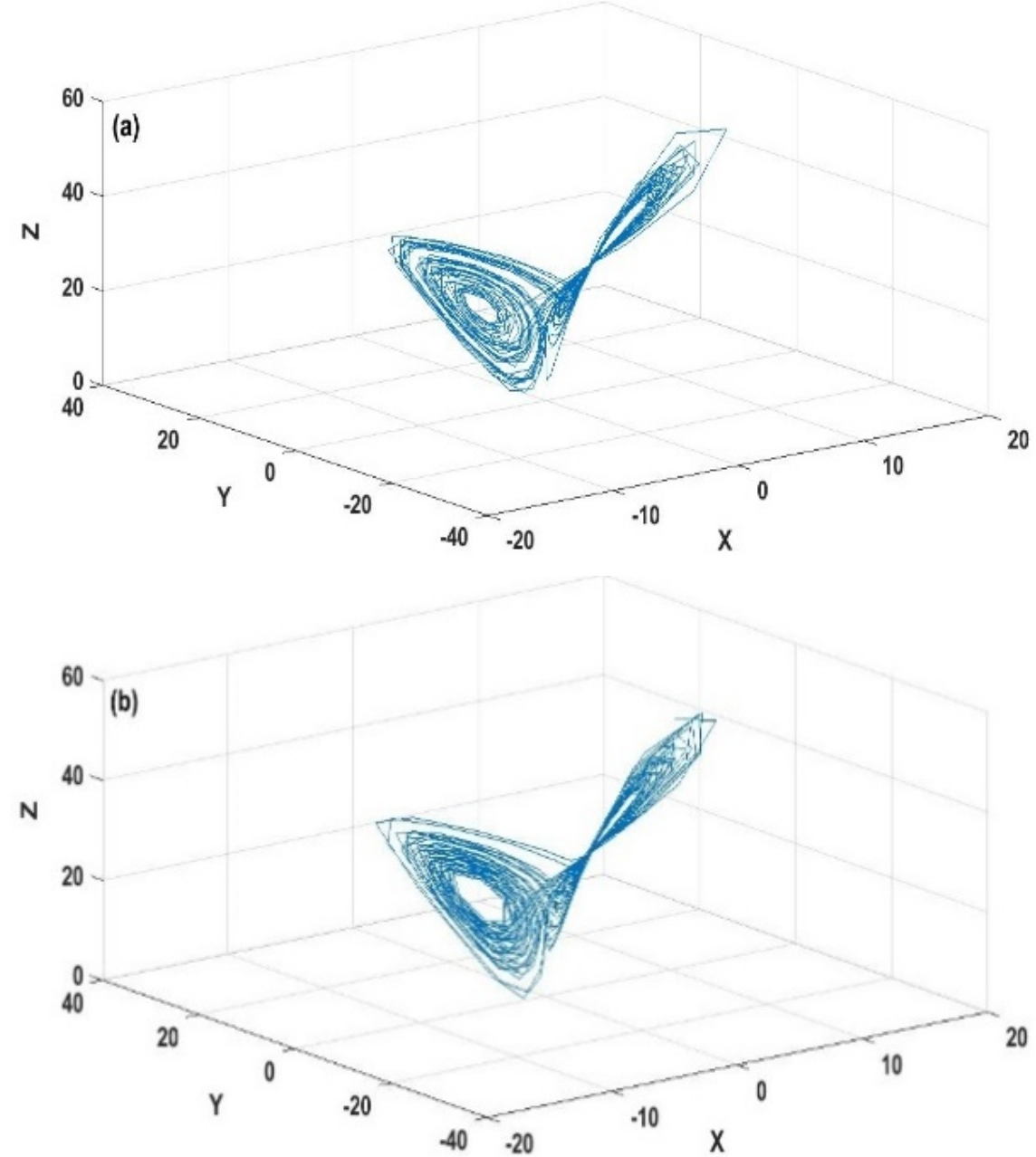

Figure 2. Trajectories in the state space of the Lorenz system with the controller (a) and without the controller (b), by initial conditions: $(0.2,0.1$ and 0.2$)$ 


\section{References}

[1] J. Ma, F. Wu, W. Jin, P. Zhou, T. Hayat, Calculation of Hamilton energy and control of dynamical systems with different types of attractors, Chaos, 27 (2017) 1-9. doi:10.1063/j.chaos. 2017.01.17.

[2] C. Barbara, C. Silvano, Hyperchaotic behavior of two bidirectional Chua's circuits, Chaos, 27 (2002) 625-637. doi: 10.1002/cta.213 / Circuit Theory Appl. 2002.04.24.

[3] G. Grassi, S. Mascolo, Synchronizing high dimensional chaotic systems via eigenvalue placement with application to cellular neural networks, Int. J. Bifurc. Chaos. 9 (1999) 705-711. doi: 10.1142/S0218127499000493 / Chaos. 1999.01.04.

[4] R. Vicente, J. Daudén and R. Toral, Analysis and characterization of the hyperchaos generated by a semiconductor laser subject, IEEE. 41 (2005) 541-548. doi: 10.1109/JQE.2005.843606 / Quantum Electron. 2005.03.24.

[5] Shaohua Luo, Quanping Sun, and Wei Cheng, Chaos control of the micro-electro-mechanical resonator by using adaptive dynamic surface technology with extended state observer, AIP, 6 (2016) 5019. Doi: 10.1063/1.4946785/ AIP Advances. 2016.05 .12

[6] P. Sriramalakshmi, A. Kavitha, P. Sanjeevikumar, Tole Sutikno, Pandav Kiran Maroti, Vigna K. Ramachandaramurthy, Control of Chaos in a Current Mode Controlled Buck Boost Converter Using Weak Periodic Perturbation Method, IJPEDS, 8(2017) 14671480. Doi: 10.11591/ijpeds.v8i4.pp1467-1480/ Power Electronics and Drive System. 2017.06.04

[7] M Fuhong, W Yaoda, G Peng, W Enrong, and J A. Auth, Bifurcations, chaos and adaptive backstepping sliding mode control of a power system with excitation limitation, AIP, 6(2016) 21. Doi: 10.1063/1.4961696/ AIP Advances/ 2016/08/12

[8] P Singh, M Kshetrimayum, B Roy, Chaos control in biological system using recursive backstepping sliding mode control, EPJST, 21 (2018) 731-746. Doi: 10.1140/epjst/e2018-800023-6/ 2018.07.18

[9] S Mobayen, F Tchier, Synchronization of a class of uncertain chaotic systems with Lipschitz nonlinearities using state-feedback control design: a matrix inequality approach, AJOF, 24(2018) 1-15/ doi: 10.1002/asjc.1512/ Asian Journal of Control. 2018.01.15

[10] A. N. Njah, V Uchechukwu, Control and synchronization of chaos in nonlinear gyros via backstepping design, JNS, 5(2008) 11-19/ doi: 10.1002/asjc.1512/ Nonlinear Science. 2008.08.05

[11] L Runzi, S Haipeng, Z Yanhui Zeng, Chaos Control and Synchronization via Switched Output Control Strategy, HINDAWI, 27 (2017) 1-11. doi: 10.1155/2017/6125102 / Complixity. 2017.11.03.

[12] C Wang, H Zhang, W Fan, M Ping, Adaptive control method for chaotic power systems based on finite-time stability theory and passivity-based control approach, Elsevier, 112 (2018) 159-167. 1doi: 0.1016/j.chaos.2018.05.005

[13] R Pérez, J Moreno, A novel Lyapunov-based trajectory tracking controller for a quadrotor: Experimental analysis by using two motion tasks, ELSEVIER, 61(2019)58-68, doi:10.1016/j.mechatronics.2019.05.006 / Decision and Control. 2019.11.04.

[14] C. Aguilar, J. Moreno, Model reference adaptive control for the trajectory tracking of a DC motor with pendular load, IASTED, 13(2013) 266-272 / doi: 10.2316/P.2013.807-038 / Intelligent Systems and Control. 2013.09.13.

[15] X Xiaojian, S Mobayen, H Ren, S Jafari, Robust finite-time synchronization of a class of chaotic systems via adaptive global sliding mode control, VIB, 24(2017) 3842-3854/ doi: 10.1177/1077546317713532/ Vibration and Control. 2017.05.06

[16] R Song, Q Wei, Chaotic system optimal tracking using data-based synchronous method with unknown dynamics and disturbances, Elsevier, 26 (2017) 73-89 .doi: 10.1088/1674-1056/26/3/030505/ Chinese Physical Society.2017.05.05

[17] J Moreno, Lyapunov function-based adaptive chaos anti control of robot manipulators, IEEE, 23(2014) 41-53, doi: 10.1109/ISIE.2014.6864788/ International Symposium on Industrial Electronics. 2014.07.28.

[18] H. Zargarzadeh, M.R. Jahed Motlagh, Anti-control of chaos in rigid motion using an internal torque source, IFAC, 23(2009) 349353, doi: 10.3182/20090622-3-UK-3004.00065/ Analysis and Control of Chaotic Systems. 2009.05.20.

[19] J. Moreno, Model reference adaptive control for the trajectory tracking of a DC motor with pendular load, IASTED, 18(2013)1-11 / doi: 10.1016/j.cnsns.2012.06.003/ Communications in Nonlinear Science and Numerical Simulation. 2013.01.23.

[20] Y Xinghuo, C Guanrong, S Yanxing, C Zhenwei, X Yang, A generalized OGY method for controlling higher order chaotic systems, IEEE, 39 (2002) 21-33, Doi: 10.1080/10260220290013507/ Decision and Control. 2002.04.01.

[21] J. Ma, F. Wu, W. Jin, P. Zhou, T. Hayat, Calculation of Hamilton energy and control of dynamical systems with different types of attractors, Chaos, 27 (2017) 1-9. doi:10.1063/j.chaos. 2017.01.17

[22] Sarasola C, Torrealdea FJ, D'Anjou A, Mouj-ahid A, Graña M, Ping Zhou and Tasawar H, Energy balance in feedback synchronization of chaotic systems, Physical Review. 69 (2004) 11606-116019. doi: 10.1103/PhysRevE. 2004.7.30.

[23] H.Kobravi, A.Erfanian, A decentralized adaptive robust method for chaos control, Chaos,19 (2009) 1-9. doi: 10.1063/1.3183806/ Chaos. 2009.04.27

[24] S Lashkari, A Sheikhani, M Hashemi Golpayegan, A Moghimi, H Kobravi, Detection and Prediction of Absence Seizures Based on Nonlinear Analysis of the EEG in Wag/Rij Animal Model, Int Clin Neurosci, 5 (2018) 21-27. doi: 10.15171/j. Int Clin Neurosci 2018.03.15.

[25] K Lehnertz, CE Elger, Can epileptic seizures be predicted? Evidence from nonlinear time series analysis of brain electrical activity, Physical Review Letter, 95 (1998) 5019. doi: 10.1103/PhysRevLett.1998.06.12. 
[26] K Lehnertz, CE Elger, Spatio-temporal dynamics of the primary epileptogenic area in temporal lobe epilepsy characterized by neuronal complexity loss, Electroencephalography and Clinical Neurophysiology, 80 (1995) 108-17. doi: 10.1103/ clinical Neurophysiology.1995.01.05.

[27] A.M. Lopez Jimineza, C. Camacho Martinez Vara De Rey, A.R. Garcia Toress, Effect of parameter calculation in direct estimation of the Lyapunov exponent in short time series, Discrete Dynamics in Nature and Society,7 (2002) 41-53, doi: 10.1080/10260220290013507/ Dynamics in Nature. 2002.04.21. 